3 SAFETY AND OUTCOMES OF A HIGH INTENSITY EXERCISE PROGRAMME IN YOUNG PATIENTS WITH HYPERTROPHIC CARDIOMYOPATHY: THE SAFE-HCM STUDY

'Joyee Basu, 'Shruti Jayakumar, ${ }^{1}$ Christopher Miles, ${ }^{1}$ Gemma Parry-Williams, ${ }^{1}$ Hamish MacLachlan, ${ }^{2}$ Nabeel Sheikh, 'Paulo Bulleros, 'Zephryn Fanton, 'Elijah Behr, ${ }^{3} J a m i e$ O'Driscoll, 'Sanjay Sharma, 'Maria Therese Tome Esteban, 'Dimitra Nikoletou, ${ }^{1}$ Michael Papadakis. 'St George's University of London, London, UK; ${ }^{2}$ Guy's and St Thomas' NHS Foundation Trust; ${ }^{3}$ Canterbury Christ Church University

\subsection{6/heartjnl-2021-BCS.3}

Background Moderate intensity exercise training in older patients with hypertrophic cardiomyopathy (HCM) can improve functional capacity, without significant harm. However, younger patients are attracted to high intensity training (HIT) regimes. PurposeTo assess the feasibility, safety and outcomes of an individually tailored, HIT programme in young patients with HCM and to assess whether observed benefits are sustained at 6 months.

Methods Eighty patients with HCM $(45.7 y \pm 8.6)$ underwent baseline clinical and psychological assessment. Individuals were randomised to a 12-week HIT programme $(n=40)$ or usual care $(n=40)$. Baseline evaluation was repeated at 12 weeks (T12). Feasibility, safety, health and psychological benefits were assessed. At 12-weeks individuals were encouraged to continue with the frequency and intensity of physical activity (PA) achieved at the end of the cardiac rehabilitation programme. Participants in the exercise arm were invited to follow-up at 6 months (T6m).

Results The majority (83\%) of participants completed the 12week study. Reasons for refusal included travel, work and family commitments. Resource requirements were similar to other programmes. All individuals felt supported, more confident to exercise, and found educational materials clear and informative. At T12 there was no significant difference between groups in the composite arrhythmia safety outcome $(p=0.99)$. There was no significant difference between groups in episodes of non-sustained ventricular tachycardia (NSVT) $(p=0.573)$ or ectopic burden $(p=0.729)$. The indices of exercise capacity were significantly improved in the exercise compared to the control group; peak VO2 $(+3.7 \mathrm{ml} / \mathrm{kg} / \mathrm{min}$ [CI 1.1,6.3], $\mathrm{p}=0.006), \mathrm{VO} 2 / \mathrm{kg}$ at anaerobic threshold (VO2/ $\mathrm{kgAT})(+2.44 \mathrm{ml} / \mathrm{kg} / \mathrm{min}$ [CI 0.6,4.2], $\mathrm{p}=0.009)$, time to AT $(+115 \mathrm{~s}$ [CI 54.3,175.9], $\mathrm{p}<0.001)$ and exercise time (max ET) $(+108 \mathrm{~s}$ [CI 33.7,182.2], p=0.005). The exercise group also demonstrated greater reduction in systolic BP $(-7.3 \mathrm{mmHg}$ [CI -11.7,-2.8], p=0.002), BMI $(-0.8 \mathrm{~kg} / \mathrm{m} 2 \quad$ [CI-1.1,-0.4], $\mathrm{p}<0.001)$, anxiety $(-2.6[\mathrm{CI}-3.6,-1.6], \mathrm{p} \leq 0.001)$ and depression $(-1.1$ [CI $-2.0,-0.2], p=0.015)$ scores. At $\mathrm{T} 6 \mathrm{~m}$ patient reported exercise adherence was comparable to baseline PA, in 33 of 34 of the exercise group attending for follow up. Most exercise gains dissipated with the exception of time to AT $(p=0.002)$, max ET $(p=0.003), \operatorname{VO} 2 / \operatorname{kgAT}(p=0.04)$ and anxiety score $(p<0.001)$ (figure 1$)$. There were no sustained episodes of atrial or ventricular arrhythmias. The incidence of NSVT did not differ between time points $(p=0.09)$.

Conclusion A 12-week HIT programme in young patients with HCM offers considerable gains in fitness and psychological outcomes, with no increase in arrhythmic burden. Further research is still required to assess the long-term safety of high intensity exercise in the HCM population. At T6m exercise levels as well as most physiological adaptations and health benefits returned to baseline, as seen in other studies when
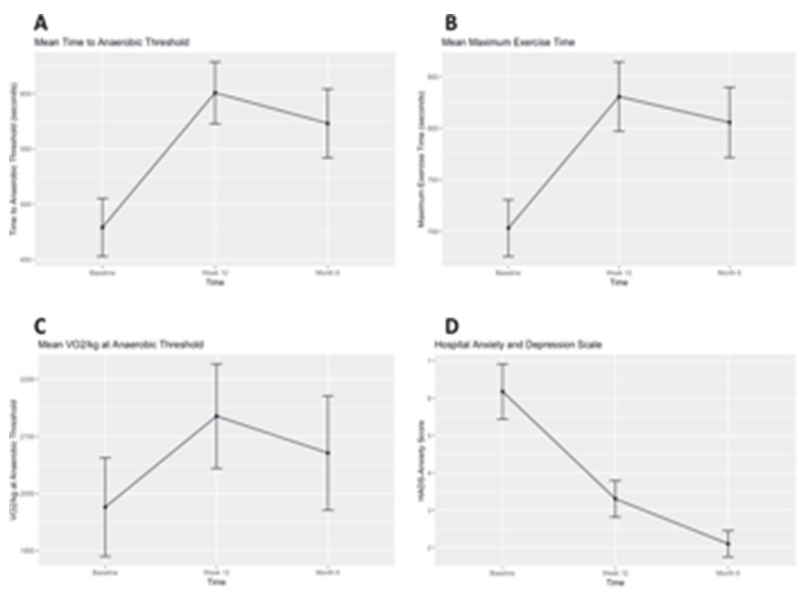

Abstract 3 Figure 1

formal participation in an exercise programme comes to an end. This highlights the importance of the implementation of strategies to encourage ongoing engagement in PA. Potential solutions include identification of barriers to exercise, as well as adoption of novel tele-rehabilitation approaches.

Conflict of Interest None

\section{$4 \quad$ IMPACT OF LEFT VENTRICULAR ASSIST DEVICE THERAPY ON SEVERE SECONDARY MITRAL REGURGITATION}

${ }^{1}$ Harish Sharma, ${ }^{2}$ Mengshi Yuan, ${ }^{1}$ Iqra Shakeel, ${ }^{2}$ Andrew Morley-Smith, ${ }^{2}$ M. Adnan Nadir, ${ }^{2}$ Colin Chue, ${ }^{3}$ Saul Myerson, ${ }^{2}$ Richard P. Steeds, ${ }^{2}$ Sern Lim. ${ }^{1}$ University of Birmingham, Birmingham, UK; ${ }^{2}$ Queen Elizabeth Hospital Birmingham; ${ }^{3}$ Department of Cardiovascular Medicine, University of Oxford

\subsection{6/heartjnl-2021-BCS.4}

Background Severe secondary mitral regurgitation (MR) worsens prognosis in patients with medically managed heart failure (HF). In patients treated by left ventricular assist device (LVAD), it is unclear whether severe MR should be corrected at time of LVAD implantation.

Purpose To evaluate impact of LVAD therapy on severe MR and non-severe MR over 1 year.

Methods Retrospective single centre study of consecutive patients who underwent Heartmate (HM) 2 or HM3 LVAD implantation between January 2011 and March 2020.

Results Of 155 patients, 20 were excluded due to LVAD exchange $(n=10)$, mitral valve repair $(n=1)$, or inaccessible pre-LVAD echocardiography $(n=9)$. Based on multiparametric grading, 29 patients had severe secondary MR and 106 nonsevere secondary MR (including none). Severe MR patients were more often female $(10 / 29$ (34\%) vs $11 / 106 \quad(10 \%)$; $\mathrm{p}=0.002)$ but were of similar age $(54 \pm 12$ vs $55 \pm 9$ years; $\mathrm{p}=0.624)$, size $(27 \pm 5$ vs $27 \pm 4 \mathrm{~kg} / \mathrm{m} 2 ; \mathrm{p}=1.0)$, with equivalent renal function $(53 \pm 22$ vs $55 \pm 20 \mathrm{ml} / \mathrm{min} / 1.73 \mathrm{~m} 2$; $\mathrm{p}=0.641$ ) and median pre-operative NT-proBNP [4076 (IQR 206-5438) vs 4914 (IQR 2706-7518) pg/L; p=0.488]. There were similar proportions of patients with ischaemic aetiology $[16 / 29(55 \%)$ vs $66 / 106(62 \%) ; p=0.488)$ and those receiving HM2 [11/29 (38\%) vs 32/106 (30\%)] and HM3 [18/29 $(62 \%)$ vs 74/106 (70\%); p=0.575] LVAD. Echocardiography before LVAD implantation demonstrated similar LV size (LV end-diastolic volume: $133 \pm 44$ vs $118 \pm 50 \mathrm{ml} / \mathrm{m} 2 ; \mathrm{p}=0.145$, LV 


\section{Non-Severe MR pre-LVAD}

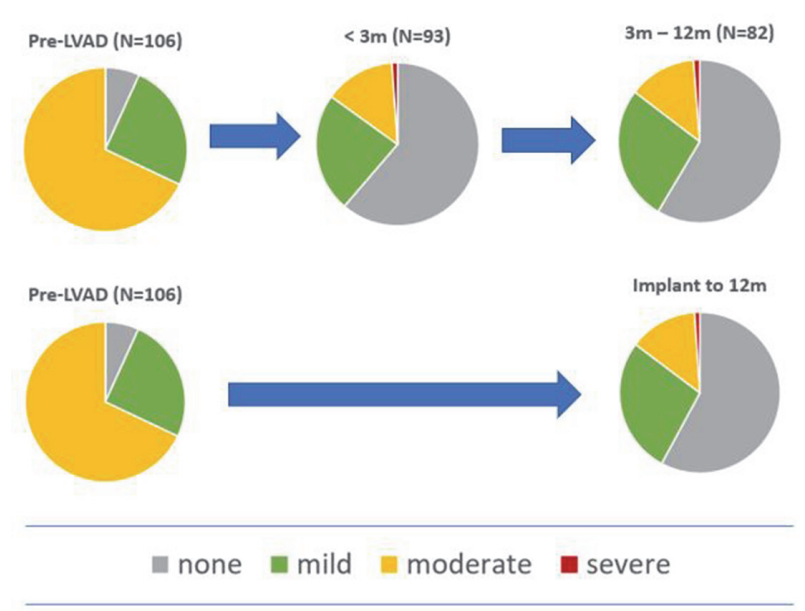

Abstract 4 Figure 1

\section{Severe MR pre-LVAD}

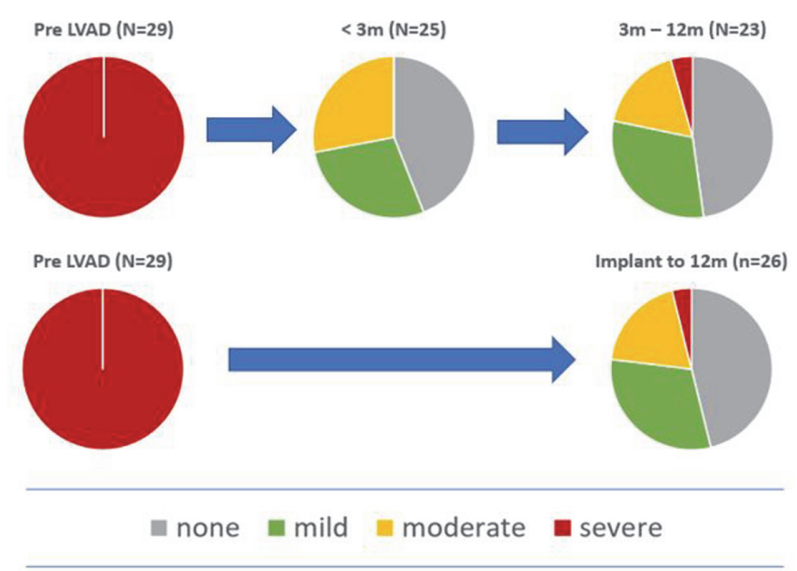

\section{Abstract 4 Figure 2}

end-systolic volume: $107 \pm 41$ vs $96 \pm 59 \mathrm{ml} / \mathrm{m} 2 ; \mathrm{p}=0.348)$ and LV ejection fraction $(17 \pm 9$ vs $17 \pm 7 \% ; \mathrm{p}=1.0)$. Severe MR patients had significantly greater $(\mathrm{p}<0.001)$ MR by proximal isovolumetric surface area $(0.93 \pm 0.27$ vs $0.60 \pm 0.16 \mathrm{~cm})$, vena contracta $(0.79 \pm 0.32$ vs $0.57 \pm 0.18 \mathrm{~cm})$, regurgitant volume $(47 \pm 25$ vs $24 \pm 12 \mathrm{ml})$, and fraction $(54 \pm 15$ vs $37 \pm 13 \%)$. Follow-up echocardiography was performed at a median 222 days (range 356 days). Patients who received cardiac transplantation were excluded. Relative severities of MR at followup were: none $=12 \quad(46 \%)$, mild $=8 \quad(31 \%)$, moderate $=5$ $(19 \%)$, severe $=1(4 \%)$ amongst patients with severe MR preLVAD, and none $=55(58 \%)$, mild $=26 \quad(27 \%)$, moderate $=13$ $(14 \%)$, severe $=1(1 \%)$ amongst patients with non-severe MR pre-LVAD (figure 1). Within $12 \mathrm{~m}$, after excluding patients who underwent cardiac transplantation (severe $M R n=4$; nonsevere MR $\mathrm{n}=2$ ), rates of HF hospitalisation [5/25 (20\%) vs $16 / 104(15 \%) ; \mathrm{p}=0.575]$ and all-cause mortality [2/25 (18\%) vs $22 / 104(21 \%) ; p=0.129)]$ were similar, irrespective of preLVAD MR severity. No patient who died during follow-up had severe MR prior to death.
Conclusion LVAD improves severe MR in 96\% of cases, resulting in 1-year rates of $\mathrm{HF}$ hospitalisation and mortality similar to patients without severe MR pre-LVAD. These data suggest mitral valve surgery at time of LVAD implantation is not warranted.

Conflict of Interest None

\section{EXAGGERATED ELASTIN TURNOVER IN CHILDHOOD AND ADOLESCENCE IN MARFAN SYNDROME - CORRELATION WITH AGE - NEW INSIGHTS FROM THE AIMS TRIAL}

'Zaid Iskandar, ${ }^{2}$ Matthew Dodd, ${ }^{3}$ Graham Stuart, ${ }^{3}$ Massimo Caputo, ${ }^{2}$ Tim Clayton, ${ }^{4}$ Calvin Chin, ${ }^{5}$ Jack Gibb, ${ }^{6}$ Anne Child, ${ }^{6}$ Jose Antonio Aragon-Martin, ${ }^{7} \mathrm{Xu}$ Yu Jin, ${ }^{8}$ Marcus Flather, ${ }^{9}$ Jeffrey T J Huang, ${ }^{1}$ Anna Maria Choy. ${ }^{1}$ Division of Molecular \& Clinical Medicine, University of Dundee, Dundee, UK; ${ }^{2}$ Clinical Trials Unit \& Dept of Medical Statistics, London School of Hygiene and Tropical Medicine; ${ }^{3}$ Bristol Heart Institute; ${ }^{4}$ National Heart Centre Singapore; ${ }^{5}$ Department of Paediatric Cardiology, Bristol Royal Hospital for Children; ${ }^{6}$ National Heart \& Lung Institute, Imperial College London; ${ }^{7}$ Oxford Heart Centre, Oxford University Hospitals NHS Foundation Trust; ${ }^{8}$ Norwich Medical School, University of East Anglia; ${ }^{9}$ School of Medicine, University of Dundee

\subsection{6/heartjnl-2021-BCS.5}

Background Marfan syndrome (MFS) is an autosomal dominant connective tissue disorder caused by mutations in the Fibrillin1 gene (FBN1) that leads to impaired elastin formation and extra-cellular matrix homeostasis. Elastin synthesis and related lamellae formation in the aorta is completed in the newborn. Thereafter further elastin turnover is thought to be minimal during life. Elastin homeostasis is little studied in patients with MFS who have been reported to have reduced elastin in their aorta and are at risk of aortic dilatation and consequent lifethreatening dissection. It is not clear if elastin loss is due to reduced elastin formation in utero or accelerated degradation during life. However, aortopathy develops early in MFS with $70 \%$ of patients developing aortic dilatation by the age of 20 . Therefore, the longitudinal and circumferential aortic growth that occurs in childhood may involve elastic lamellae remodelling that is impaired in MFS. Desmosine is the cross-link component in the elastin molecule and is exclusively released from mature elastin breakdown, thus is a physiologically relevant biomarker of elastin turnover. We hypothesize that physiologic aortic growth is associated with elastic lamellae remodelling and increased elastin turnover, detected by elevated plasma desmosine (pDES), and that elastin turnover is exaggerated during the fast-growing period in MFS.

Purpose The aims of this study were 1 . to investigate the relationship between elastin turnover and age in the control subjects, 2. to compare the elastin turnover in MFS with control subjects.

Methods pDES was measured by stable isotope dilution LCMS/MS in 113 MFS subjects (48\% male, mean age 18.2 \pm 9.4 (SD) years), mean aortic root $33.8 \pm 2.1 \mathrm{~mm}$ and Z-score 3.4 \pm 2.1 in the AIMS trial at baseline before intervention, and in 109 healthy controls (46\% male, mean age $26.1 \pm 9.5$ years).

Results pDES levels were associated positively with age, body surface area and negatively with diastolic BP in the control group ( $\mathrm{p}=0.05$ and 0.21 respectively after correcting for age). In MFS subjects pDES also positively correlated with age and male sex $(p<0.05)$. Interestingly, the age-dependent association with pDES showed a peak distribution in both control and MFS groups (figure 1) where teenage children expressed the highest pDES levels. MFS subjects had significantly higher 